POETRY

\title{
The Nature of War
}

\section{Riley Barber}

Scarlet snow blankets the field, bordered by a fence with metallic spirals on top.

Men lay on the ground, their limbs precariously angled in a silent snow angel.

Metal rain thundered down, accompanied by loud BANGS! of thunder, golden lightning brightening the sky like a mass of fireworks.

Silver birds buzzed restlessly around, surveying the scene below.

Children played in masks, chasing down the street into sheds at their parent's beck and call.

City lights turned out for the night, an eerie siren broke through the silent darkness, summoning families to safety. 